\title{
Genomic Characterization and Expressional Profiles of Autophagy-Related Genes (ATGs) in Oilseed Crop Castor Bean (Ricinus communis L.)
}

\author{
Bing Han ${ }^{1,2}$, Hui Xu ${ }^{3}$, Yingting Feng ${ }^{3}$, Wei Xu ${ }^{1}{ }^{\mathbb{C}}$, Qinghua Cui ${ }^{3, *}$ and Aizhong Liu ${ }^{4, *}$ \\ 1 Department of Economic Plants and Biotechnology, and Yunnan Key Laboratory for Wild Plant Resources, \\ Kunming Institute of Botany, Chinese Academy of Sciences, Kunming 650204, China; \\ hanbing@mail.kib.ac.cn (B.H.); xuwei@mail.kib.ac.cn (W.X.) \\ 2 University of the Chinese Academy of Sciences, Beijing 100049, China \\ 3 College of Life Sciences, Yunnan University, Kunming 650091, China; xh15969478647@163.com (H.X.); \\ fyt18087864922@163.com (Y.F.) \\ 4 Key Laboratory for Forest Resources Conservation and Utilization in Southwest Mountains of China, \\ College of Forestry, Southwest Forestry University, Kunming 650201, China \\ * Correspondence: cuiqinghua@ynu.edu.cn (Q.C.); liuaizhong@mail.kib.ac.cn (A.L.); \\ Tel.: +86-87165223125 (A.L.)
}

Received: 30 December 2019; Accepted: 14 January 2020; Published: 15 January 2020

\begin{abstract}
Cellular autophagy is a widely-occurring conserved process for turning over damaged organelles or recycling cytoplasmic contents in cells. Although autophagy-related genes (ATGs) have been broadly identified from many plants, little is known about the potential function of autophagy in mediating plant growth and development, particularly in recycling cytoplasmic contents during seed development and germination. Castor bean (Ricinus communis) is one of the most important inedible oilseed crops. Its mature seed has a persistent and large endosperm with a hard and lignified seed coat, and is considered a model system for studying seed biology. Here, a total of 34 RcATG genes were identified in the castor bean genome and their sequence structures were characterized. The expressional profiles of these RcATGs were examined using RNA-seq and real-time PCR in a variety of tissues. In particular, we found that most RcATGs were significantly up-regulated in the later stage of seed coat development, tightly associated with the lignification of cell wall tissues. During seed germination, the expression patterns of most RcATGs were associated with the decomposition of storage oils. Furthermore, we observed by electron microscopy that the lipid droplets were directly swallowed by the vacuoles, suggesting that autophagy directly participates in mediating the decomposition of lipid droplets via the microlipophagy pathway in germinating castor bean seeds. This study provides novel insights into understanding the potential function of autophagy in mediating seed development and germination.
\end{abstract}

Keywords: autophagy; castor bean; gene expression; seed development; seed germination

\section{Introduction}

Cellular autophagy is a conserved process for turning over damaged organelles or recycling cytoplasmic contents in cells. It is considered an adaptive response to intracellular and extracellular environmental changes [1]. Although most autophagy-related genes (ATGs) were initially identified in yeast (Saccharomyces cerevisiae), studies have revealed that the autophagy process occurs widely in eukaryotic organisms, involving diverse and complicated physiological processes [2,3]. Generally, the autophagy process mobilizes two autophagic pathways: macroautophagy and microautophagy [4]. Macroautophagy is the principal pathway for decomposing substrates, using 
specialized double-membrane vesicles called autophagosomes for capturing materials [5], whereas microautophagy is a nonselective degradative process in which degradative materials are directly engulfed by lysosomes in mammals or vacuoles in plants [6]. Different ATGs carry out diverse functions at different stages of autophagy. Major components involved in autophagy include the ATG1/ATG13 kinase complex (that responds to nutritional cues provided by TOR, a conserved Ser/Thr kinase that regulates cell growth and metabolism), the ATG9/2/18 transmembrane complex (that supplies membranes to the autophagosome), the PI3 (phosphatidylinositol-3) kinase complex (that promotes vesicle nucleation), and the ATG8/ATG12 conjugation system (that assists with vesicle expansion and closure) [2]. In mammals, the autophagy process is functionally involved in regulating cell differentiation, cell senescence and apoptosis, further activating cellular response to starvation and adjusting the cell cycle [1].

In plants, studies have found that autophagy is broadly involved in diverse biological processes including metabolic nutrient recycling, leaf development and senescence [7]. Loss-of-function mutations of AtATG8s and AtATG18s have given rise to an induced expression of AtSEN1 (a senescence marker gene) and led to the occurrence of leaf senescence and yellowing in Arabidopsis, suggesting that the autophagy process is critical in regulating leaf senescence and cell apoptosis in plants $[8,9]$. Many studies found that some ATGs (such as ATG4) were functionally required in mediating cell differentiation and elongation in Arabidopsis root tissues [10-12]. ATG6 was functionally involved in regulating pollen vigor and germination $[13,14]$. There was additional evidence that plant cells cultured in vitro under nutrient starvation could produce autophagosomes to decompose cytoplasmic materials in vacuoles [15].

Autophagic-like pathways are also considered to be critical channels for transporting materials to the vacuole at different stages of plant development. Studies have demonstrated that autophagy is involved in regulating the accumulation of storage reservoirs (such as seed production) by altering nitrogen use efficiency in Arabidopsis and rice [16,17]. In tobacco [18] and maize [19], most ATGs were up-regulated in the late stage of developing seeds, implying that autophagy might have a role in the regulation of seed maturation. However, very little is known about the specific role of autophagy in this process. In addition, studies have found that autophagy can play a critical role in the decomposition of lipid droplets during the mobilization of fat (triacylglycerols) metabolism by sequestering a discrete region of the lipid droplets within animal cells. This selective autophagy specifically involved in lipid decomposition is called lipophagy [20,21]. Similar to the structure of lipid droplets, an oil body is the organelle for storing lipids within plant cells. There was evidence that showed lipophagy occurred in rice pollen maturation by decomposing oil bodies and providing energy for pollen development [22]. Usually, for oilseeds, the mobilization of storage oils mainly occurs in relation to seed germination in which storage oils are rapidly decomposed to release energy for activating seedling growth. However, whether the lipophagy process is required in the mobilization of storage oils in oilseeds remains unknown.

Although the genomes of many plants have been completely sequenced and ATG genes are easy to identify, ATG genes have only been identified in a few plants, such as Arabidopsis thaliana [23-26], rice [27], tobacco [18], maize [19], pepper [28] and grape [29]. Further investigations on the identification and characterization of $A T G s$ at the genome level from diverse species are necessary to increase our understanding of ATGs in plants. Castor bean (Ricinus communis L., Euphorbiaceae) is one of the most important inedible oilseed crops and its seed oil is widely used in industry, especially for producing lubricating oil and biodiesel [30-32], because of its high content of the unique fatty acid ricinoleic acid. Although endosperm is unusual in dicots, castor bean is a typical member of this unusual group [33]. Thus, compared with Arabidopsis thaliana, castor bean is often considered a model system for studying seed biology of endospermous dicots [34-36]. In this study, based on the available genome data of castor beans, we identified all castor bean ATG genes, then characterized their structures and analyzed their expression over the castor bean life cycle. According to the expressional profiles of ATGs among different tissues, we found that autophagy might be required during seed development (in particular, 
seed coat development) and seed germination (the decomposition of storage oils in endosperm). This study provides a comprehensive profile of ATGs and their specific expression profiles among different tissues (in particular, for developing and germinating seeds) in castor beans, which adds to our understanding of the potential functions of autophagy during seed development and germination.

\section{Results}

\subsection{Identification of Genes Encoding ATG Proteins in Castor Bean}

Thirty-four putative RcATGs (Table 1) were identified by bioinformatic analyses of the published castor bean genomic sequences [37]. The 34 putative RcATGs vary from 115 aa to 1989 aa in length, from $13.2 \mathrm{kDa}$ to $218.4 \mathrm{kDa}$ in molecular weight and from 4.79 to 9.59 in protein isoelectric point (IP). To inspect the validation of the putative RcATGs, each RcATG amino acid sequence was checked in Pfam to test whether the conserved ATG domains were present in the RcATG sequence. Results showed that all RcATGs were validated. The 34 RcATGs were named and classified into 18 ATG families following the Arabidopsis category and nomenclature criteria [23-26] as listed in Table 1. Of them, RcATG1 and RcATG13 include three members (RcATG1a, RcATG1b and RcATG1c; RcATG13a, $R c A T G 13 b$ and RcATG13c), respectively; both RcATG18 and RcATG8 have seven members (RcATG18 $a-g ;$ RcATG $8 a-g)$; and other RcATGs have only a single member. Based on the autophagy process pathway, the 34 RcATGs were predicted to participate in the different processes of autophagy. In castor beans, key catalytic enzymes that cover each necessary step in the autophagy pathways were identified, including induction of initiation for the ATG1/13 kinase complex (such as RcATG1 and RcATG13) and PI3 kinase complex (such as RcATG1, RcVPS15 and RcVPS34), membrane transport (such as the RcATG9, RcATG2 and RcATG18 complex) and elongation of autophagosome (such as the RcATG8 and RcATG12 conjugation system). These catalytic enzymes are essential for the occurrence of autophagy.

Table 1. The ATGs in castor beans.

\begin{tabular}{|c|c|c|c|c|c|c|c|}
\hline Gene & $\begin{array}{l}\text { Arabidopsis } \\
\text { ID }\end{array}$ & Gene & $\begin{array}{l}\text { Castor } \\
\text { Bean ID }\end{array}$ & $\begin{array}{l}\text { No. of Amino } \\
\text { Acid Residues }\end{array}$ & $\operatorname{Mw}(k D a)$ & PI & $\begin{array}{l}\text { E-value to } \\
\text { Arabidopsis }\end{array}$ \\
\hline \multicolumn{8}{|c|}{ ATG1/13 Kinase Complex } \\
\hline AtATG1a & AT3G61960 & RcATG1a & 30076.m004465 & 676 & 75.1 & 5.90 & $4.4 \times 10^{-66}$ \\
\hline AtATG1b & AT3G53930 & RcATG1b & 29973.m000397 & 694 & 76.9 & 6.44 & $7.6 \times 10^{-62}$ \\
\hline AtATG1c & AT2G37840 & & & & & & \\
\hline AtATG1t & AT1G49180 & RcATG1t & 29659.m000143 & 321 & 36.6 & 8.29 & $1.2 \times 10^{-88}$ \\
\hline AtATG13a & AT3G49590 & RcATG13a & 30098.m001725 & 621 & 69.4 & 8.81 & $3.5 \times 10^{-103}$ \\
\hline \multirow[t]{2}{*}{ AtATG13b } & AT3G18770 & RcATG13b & 30206.m000754 & 342 & 39.5 & 9.59 & $3 \times 10^{-83}$ \\
\hline & & RcATG13c & 30206.m000753 & 220 & 23.9 & 5.42 & $2.1 \times 10^{-26}$ \\
\hline AtATG11 & AT4G30790 & RcATG11 & 30114.m000524 & 1145 & 129.2 & 5.73 & 0 \\
\hline AtATG101 & AT5G66930 & RcATG101 & 29676.m001649 & 205 & 24.1 & 5.94 & $7.9 \times 10^{-76}$ \\
\hline \multicolumn{8}{|c|}{ PI3 Kinase Complex } \\
\hline AtVPS34 & AT1G60490 & RcVPS34 & 29631.m001016 & 813 & 93 & 6.48 & 0 \\
\hline AtATG6 & AT3G61710 & RcATG6 & 29742.m001430 & 523 & 59.9 & 5.85 & $2.1 \times 10^{-201}$ \\
\hline AtVPS15 & AT4G29380 & RcVPS15 & 29912.m005424 & 1455 & 163.5 & 7.00 & 0 \\
\hline \multicolumn{8}{|c|}{ ATG9/2/18 Complex } \\
\hline AtATG2 & AT3G19190 & RcATG2 & 29801.m003197 & 1989 & 218.4 & 5.43 & 0 \\
\hline AtATG9 & AT2G31260 & RcATG9 & 29584.m000241 & 864 & 99.7 & 6.32 & $8.4 \times 10^{-301}$ \\
\hline AtATG18a & AT3G62770 & RcATG18a & 30129.m000356 & 447 & 49 & 6.59 & $3.6 \times 10^{-32}$ \\
\hline AtATG18b & AT4G30510 & RcATG18b & 28612.m000123 & 349 & 38 & 8.86 & $7.1 \times 10^{-146}$ \\
\hline AtATG18c & AT2G40810 & RcATG18c & 29729.m002396 & 598 & 67 & 7.56 & $1.2 \times 10^{-81}$ \\
\hline AtATG18d & AT3G56440 & RcATG18d & 30128.m008811 & 330 & 36.6 & 9.33 & $1.6 \times 10^{-70}$ \\
\hline AtATG18e & AT5G05150 & RcATG18e & 28166.m001060 & 891 & 96.9 & 6.44 & 0 \\
\hline AtATG18f & AT5G54730 & RcATG18f & 29703.m001515 & 1016 & 110.7 & 6.25 & $3 \times 10^{-115}$ \\
\hline AtATG18g & AT1G03380 & RcATG18g & 30128.m008700 & 991 & 107.6 & 5.44 & $1 \times 10^{-113}$ \\
\hline AtATG18h & AT1G54710 & & & & & & \\
\hline
\end{tabular}


Table 1. Cont.

\begin{tabular}{|c|c|c|c|c|c|c|c|}
\hline Gene & $\begin{array}{l}\text { Arabidopsis } \\
\text { ID }\end{array}$ & Gene & $\begin{array}{c}\text { Castor } \\
\text { Bean ID }\end{array}$ & $\begin{array}{l}\text { No. of Amino } \\
\text { Acid Residues }\end{array}$ & Mw(kDa) & PI & $\begin{array}{l}\text { E-value to } \\
\text { Arabidopsis }\end{array}$ \\
\hline \multicolumn{8}{|c|}{ ATG8/12 Conjugation System } \\
\hline AtATG3 & AT5G61500 & RcATG3 & 30147.m014252 & 314 & 35.5 & 4.79 & $3.2 \times 10^{-150}$ \\
\hline AtATG4a & AT2G44140 & RcATG4 & 28966.m000547 & 489 & 53.9 & 5.13 & $2.3 \times 10^{-140}$ \\
\hline AtATG4b & AT3G59950 & & & & & & \\
\hline AtATG5 & AT5G17290 & RcATG5 & 30171.m000414 & 368 & 41.9 & 5.28 & $3.2 \times 10^{-115}$ \\
\hline AtATG7 & AT5G45900 & RcATG7 & 29900.m001609 & 710 & 77.9 & 5.35 & $3.3 \times 10^{-235}$ \\
\hline AtATG8a & AT4G21980 & RcATG8a & 29842.m003584 & 120 & 13.8 & 7.88 & $3.7 \times 10^{-53}$ \\
\hline AtATG8b & AT4G04620 & RcATG8b & 30064.m000482 & 125 & 14.3 & 7.89 & $5.5 \times 10^{-52}$ \\
\hline AtATG8c & AT1G62040 & RcATG8c & 30064.m000481 & 120 & 13.9 & 7.89 & $7 \times 10^{-52}$ \\
\hline AtATG8d & AT2G05630 & RcATG8d & 29636.m000773 & 122 & 14.1 & 7.85 & $5.8 \times 10^{-48}$ \\
\hline AtATG8e & AT2G45170 & RcATG8e & 30174.m008829 & 117 & 13.5 & 8.75 & $1.2 \times 10^{-45}$ \\
\hline AtATG8f & AT4G16520 & RcATG8f & 30076.m004580 & 125 & 14.5 & 6.73 & $1.2 \times 10^{-33}$ \\
\hline AtATG8g & АT3G60640 & RcATG8g & 29889.m003380 & 115 & 13.2 & 9.30 & $2 \times 10^{-31}$ \\
\hline AtATG8h & АТЗG06420 & & & & & & \\
\hline AtATG8i & AT3G15580 & & & & & & \\
\hline AtATG10 & АT3G07525 & RcAtg10 & 30178.m000879 & 233 & 26.9 & 5.25 & $7.3 \times 10^{-57}$ \\
\hline AtATG12a & AT1G54210 & RcAtg12 & 29706.m001289 & 154 & 17.4 & 8.58 & $1.3 \times 10^{-20}$ \\
\hline AtATG12b & АT3G13970 & & & & & & \\
\hline AtATG16L & AT5G50230 & RcATG16L & 30147.m013828 & 520 & 57.6 & 6.06 & $1.3 \times 10^{-203}$ \\
\hline
\end{tabular}

The sequence similarities of each homologous gene between castor beans and Arabidopsis were further analyzed, resulting in the high similarity of ATGs in sequence between castor beans and Arabidopsis. As shown in Figure 1, the gene structures of orthologous genes, including the distribution, number, length and splicing phase of intron/exon organization, are highly conserved between Arabidopsis and castor beans, although the intron length of some RcATGs varies, such as in ATG2, ATG3, ATG4, ATG6, ATG9, ATG10, ATG11 and ATG16L. We also noted that the number of exons or introns for ATG7, ATG12 and VPS15 vary between species, which suggested that different alternative splicing existed in Arabidopsis and castor beans. For the ATG1, ATG8, ATG13 and ATG18 subfamilies, most members shared similar gene structure, despite slight variation in the number of exons or introns. These results suggest that the gene structures of ATGs were highly conserved during plant evolution.

To further understand the phylogenetic relationships of RcATGs, we conducted phylogenetic analyses in the multi-member subfamilies ATG1, ATG13, ATG18 and ATG8, using the amino acid sequences of RcATGs and their orthologs from Arabidopsis [23-26], rice [17], tobacco [18], maize [19] and yeast (S. cerevisiae) [3]. As shown in Figure 2, in the ATG1 subfamily, ATG1s were divided into two clades according to their evolutionary relationship (Figure 2A). The motif distribution in clade I is very similar compared to clade II. In the ATG13 subfamily, ATG13s were divided into three clades according to their sequence similarity (Figure 2B). Interestingly, RcATG13c was placed outside the clades because it did not contain many of the diagnostic motifs. In the ATG18 subfamily, ATG18s were clustered into four clades. Proteins in clade III and clade IV have more motifs compared with the other two clades (Figure 2C). In the ATG8 subfamily, the generated phylogenetic tree formed two distinct clades with well-supported bootstrap values (Figure 2D). It was clearly observed that almost all ATG8s were similar in length and had identical numbers of motifs, indicating that ATG8s were quite conserved across different species. However, 4 out of 6 ATG8s in clade II (66.7\%) lack one or two motifs, whereas only ZmATG8e (4\%) in clade I is absent. 


\section{ATG1 kinase complex}

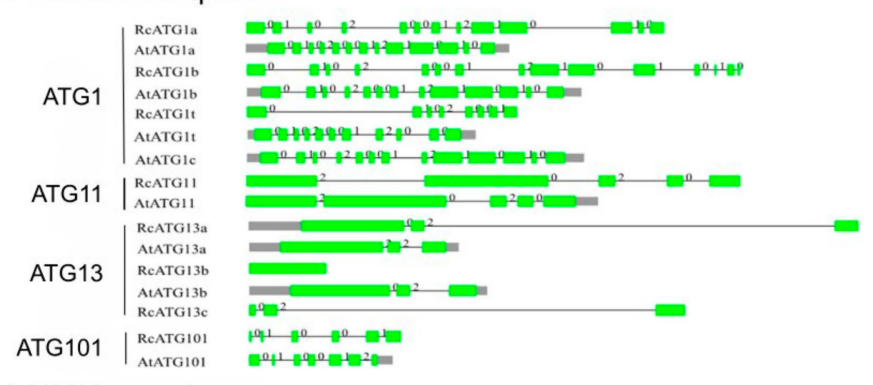

ATG2/9/18 complex

ATG2 | $\begin{aligned} & \text { RcATG2 } \\ & \text { ALATG2 }\end{aligned}$

ATG9 | $\begin{aligned} & \text { RcATG9 } \\ & \text { AtATG9 }\end{aligned}$

ReATG18a

AtATGI8a

ReATG18b

ALATCIS

RcATGise

RCATGI8

ATG18

ReATGI8d

ReATG18

ALATG18

ReATG18f

ALATG I8f

ReATG18g

ALATG18,

$\mathrm{PI} 3$ kinase complex
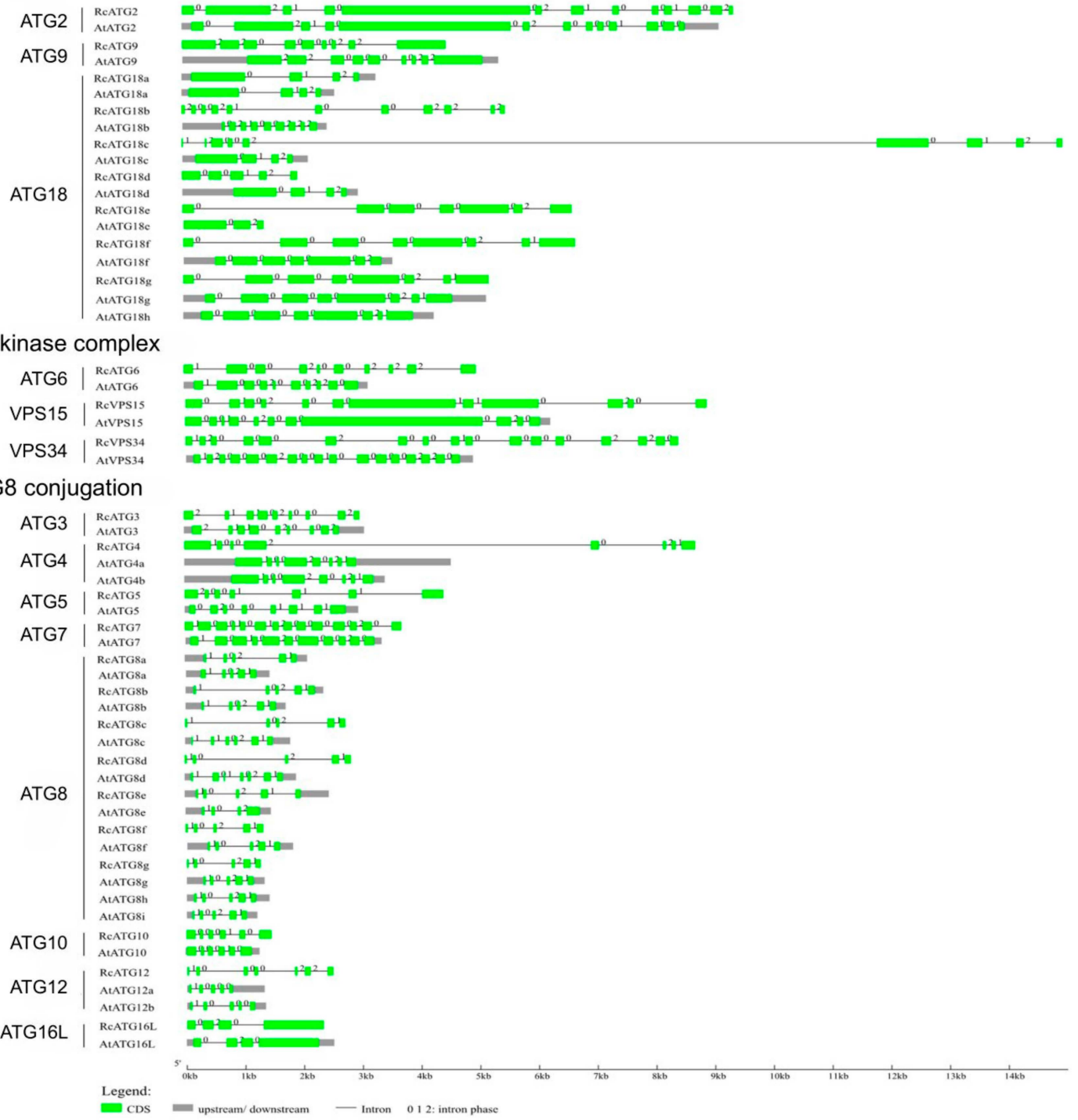

Figure 1. Exon/intron organizations of AtATGs and RcATGs. Green boxes indicate the exon regions and black lines indicate introns. The splicing phases: 0 , splicing occurred after the third nucleotide of the codon; 1 , splicing occurred after the first nucleotide of the codon; 2 , splicing occurred after the second nucleotide of the codon. 

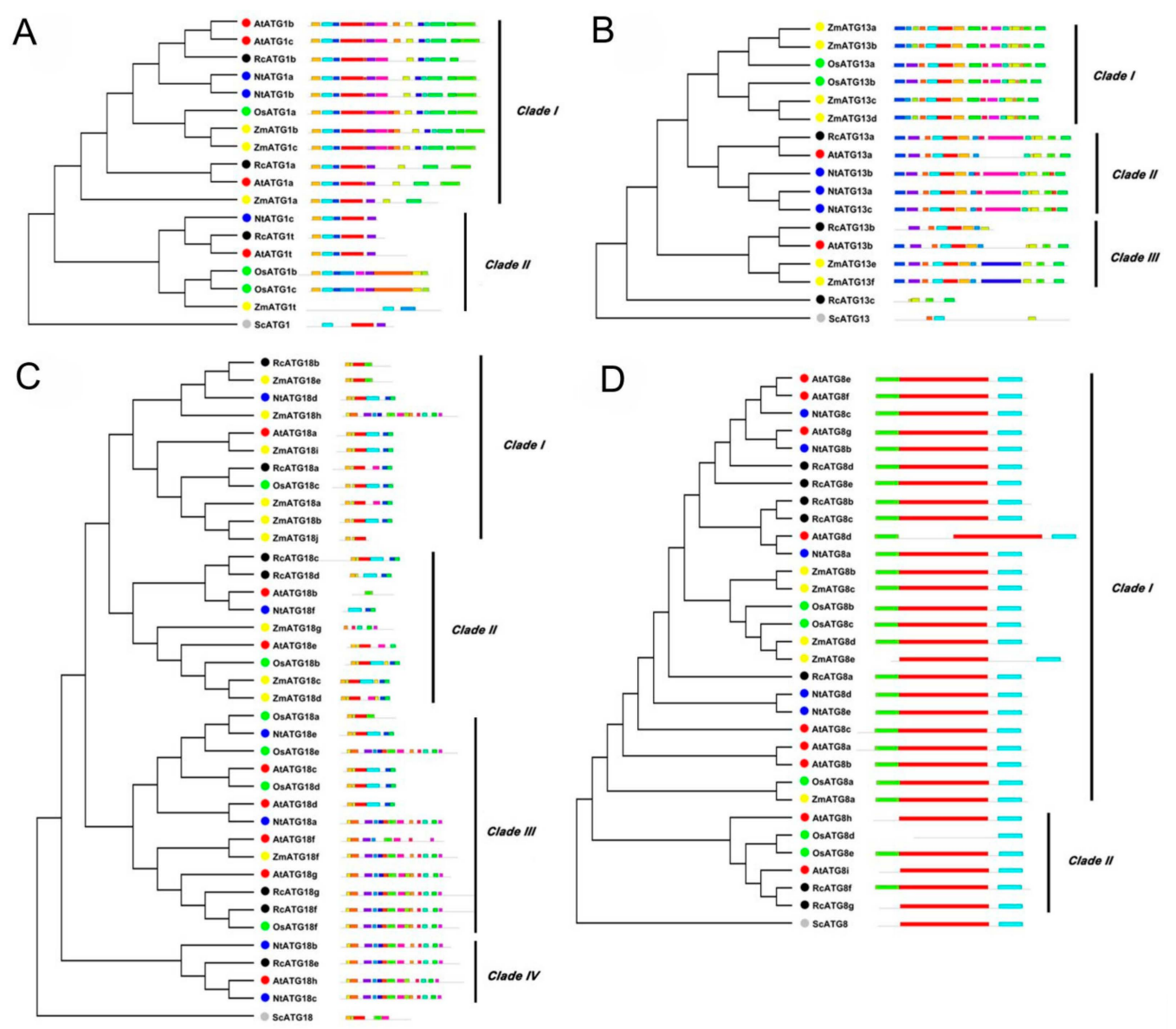

Figure 2. Bioinformatics analysis of autophagy subfamily genes in different species (i.e., Rc, Ricinus communis L.; At, Arabidopsis thaliana; Os, Oryza sativa; Nt, Nicotiana tabacum; Zm, Zea mays; Sc, Saccharomyces cerevisiae). (A) Phylogenetic tree and motif analysis of the ATG1 subfamily. (B) Phylogenetic tree and motif analysis of the ATG13 subfamily. (C) Phylogenetic tree and motif analysis of the ATG18 subfamily. (D) Phylogenetic tree and motif analysis of the ATG8 subfamily.

\subsection{Expression Profiles of RcATGs in Various Tissues}

Although the occurrence of the autophagy process requires the participation of multiple members within an activated autophagy network, a number of studies have shown that the expressional profiles of many ATG genes varied among different tissues [19], implying that different ATG genes might be involved in different biological processes. To understand the potential functions of RcATGs, we investigated the expression profiles of RcATGs in different tissues, including root, stem, leaf, seedling, ovule, capsule, inflorescence and seed across different development stages (seed1-seed5). The FPKM values of different tissues were acquired from the castor bean genome database (https: //woodyoilplants.iflora.cn/). As shown in Figure 3, most RcATGs were differentially expressed among tissues. For instance, RcATG10, RcATG18g and RcATG8a were more highly expressed in root relative to other tissues; RcATG1a, RcATG8c, RcATG101, RcATG18b, RcATG7, RcATG5 and RcATG16L were more highly expressed in reproductive tissues (ovule, capsule and inflorescence) and early seed (seed1 and seed2); RcATG18d and RcATG13c were more highly expressed in seed 4 and seed2, respectively. During seed development, fewer genes were preferentially expressed in late seeds (seed3-seed5), especially fully mature seeds (seed5), while many ATGs were highly expressed in early seeds (seed1 and seed2). Depending on the gene expression pattern, hierarchical cluster analysis was performed to further explore similarities among samples. Twelve samples clustered into two major clades, designated as clade I and clade II. Clade II bifurcated into two subclades; clade IIa corresponded to vegetative tissues including root, leaf and stem; and clade IIb corresponded to early seed tissues (seed1 
and seed2) and reproductive tissues, such as ovule, capsule and inflorescence. Clade I included all mature seed tissues, including seed3, seed 4 and seed5. These results suggested that autophagy may not only vary greatly among different tissues but also participate in different biological processes at different seed development stages in castor beans.
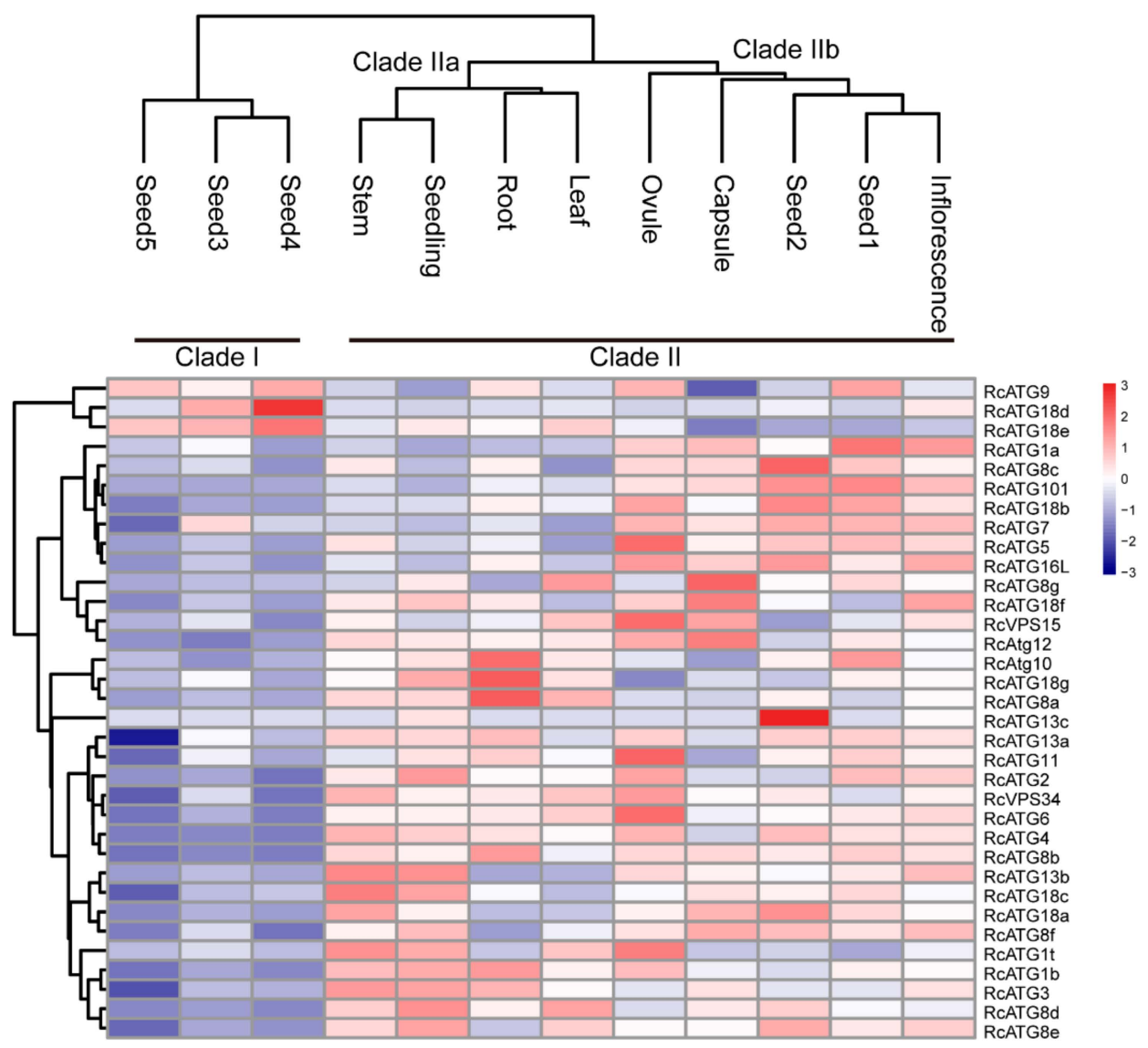

Figure 3. Expression profiles of RcATGs among different tissues. Blue box indicates the lower expression level of RcATGs, whereas the red box indicates the higher transcriptional level of RcATGs. The scale bar represents the relative expression level after normalization. Seed1-5 represent seeds at different development stages. The analysis was based on RNA-seq data downloaded from the castor bean genome database (https://woodyoilplants.iflora.cn/). Based on the 'complete' clustering method, hierarchical cluster analysis was performed.

\subsection{Expression Profiles of ATGs in Developing Seeds}

Unlike most dicotyledonous plants (such as Arabidopsis), the endosperm of the castor bean is persistent through seed development. After nuclear endosperm cells form cell walls and seed coats rapidly disappear, the endosperm cells divide quickly and eventually form a large nutritive tissue [36].

To investigate how autophagy was functionally involved in mediating seed development in castor beans, the expressional profiles of RcATGs were inspected by RT-qPCR in embryo, endosperm and seed coat tissues during seed development. As it is difficult to isolate endosperm and embryo tissues until about 20 or $25 \mathrm{DAP}$, we sampled the developing endosperm between 20 and $50 \mathrm{DAP}$, developing the embryo between 25 and 40 DAP. Because the seed coat will disappear after 30 DAP, we sampled the developing seed coat between 10 and 30 DAP. As shown in Figure 4, several RcATGs such as RcATG1t, RcATG18d and RcATG18e were highly expressed in developing endosperm tissues, while most RcATGs were not expressed or slightly expressed in developing embryo tissues. Interestingly, the expression levels of most RcATGs increased with seed coat development. Moreover, a large number of RcATGs were highly expressed in the late stage of seed coat development, in particular at the stage of 
25-30 DAP, when the seed coat cellular tissue disappeared rapidly and was fiberized (see Figure S1). The disappearance of the seed coat, considered a programmed apoptosis [38], provides space for the formation of the castor bean endosperm.

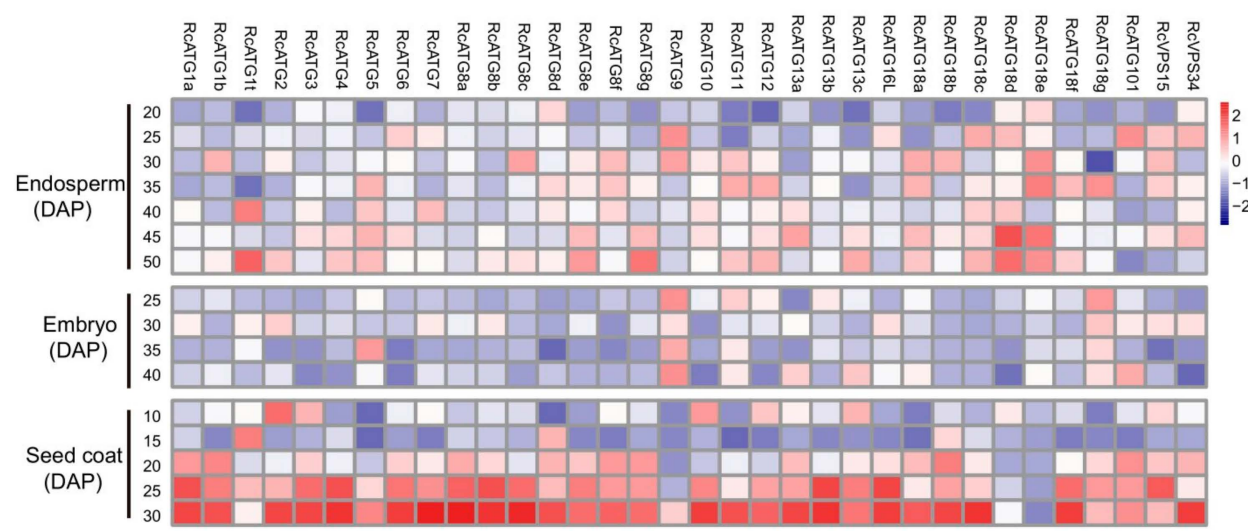

Figure 4. Expression profiles of RcATGs in castor bean seeds. The expression profile was constructed depending on the relative expression level of RcATGs in different materials. The expression is normalized to RcACTIN2 and the data are means \pm S.D. from three biological replicates. Blue boxes indicate the lower transcriptional level of RcATGs, whereas red boxes indicate the higher expression level of RcATGs. The scale bar represents relative expression level after normalization. DAP: days after pollination.

\subsection{Expression and Involvement of Autophagy in Mediating Storage Lipid Decomposition during Seed} Germination

Increasing evidence shows that lipophagy is required for the decomposition of cellular lipid droplets $[39,40]$. However, little is known about whether lipophagy is involved in the decomposition of oil bodies during oilseed germination. To examine whether autophagy is involved in storage lipid decomposition during castor bean germination, we first inspected the change of oil content in seed germination. As shown in Figure 5, storage lipids were rapidly degraded starting 3 DAG. Upon examining the expression profiles of RcATGs by RT-qPCR in endosperms during castor bean seed germination, we found that most genes were not expressed (or marginally expressed) within the first two days and began to be up-regulated 3 DAG (Figure 6A), which is closely associated with the decomposition trend of storage lipids in seed germination (Figure 5). These results suggested that autophagy might have a role in mediating the decomposition of storage lipids in germinating castor bean seeds.

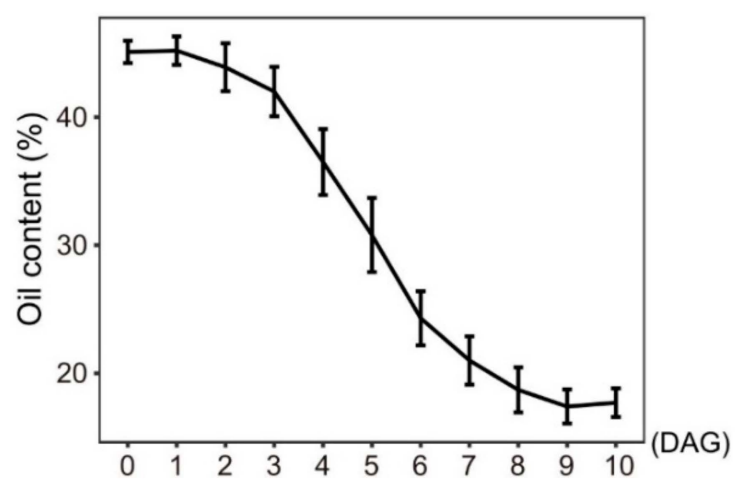

Figure 5. Oil content of germinating endosperm. DAG: days after germination. Bars $=$ means \pm SD from three biological replicates. 


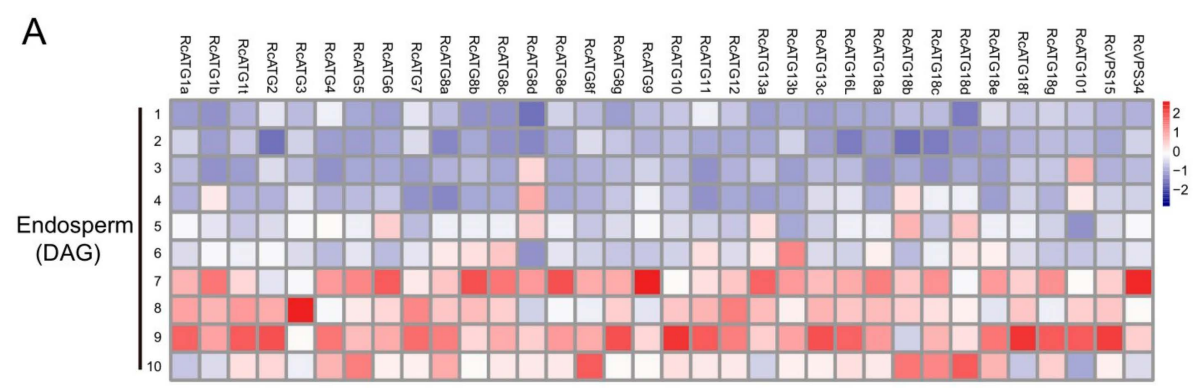

B

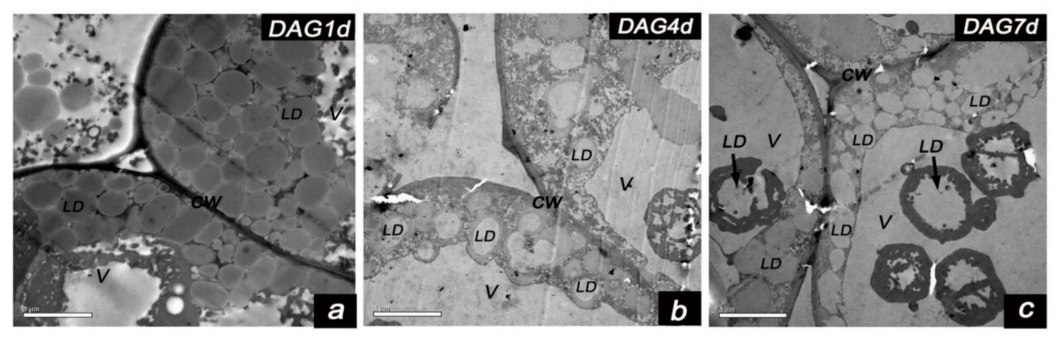

Figure 6. (A) Expression profiles of RcATGs in germinating endosperm. The expression profile was constructed depending on the relative expression level of RcATGs at different germination stages (DAG: days after germination). The expression is normalized to RcACTIN2 and the data are means \pm S.D. from three biological replicates. Blue boxes indicate the lower transcriptional level of RcATGs and red boxes indicate the higher expression level of RcATGs. The scale bar represents relative expression level after normalization. (B) Transmission electron microscopy of germinating endosperm (LD: lipid droplets; V: vacuole; CW: cell wall). a: DAG1d, b: DAG4d, c: DAG7d.

As mentioned above, lipophagy is involved in the breakdown of LDs for the maintenance of lipid homeostasis [40]. Usually, lipophagy occurs via two different pathways. One, termed microlipophagy, is where the LDs are directly degraded into fatty acids inside vacuoles. This has been observed in lower organisms such as single cell algae (Auxenochlorella protothecoides) and yeast (S. cerevisiae) [41,42]. The second is termed macrolipophagy, in which the degradation of LDs are mediated by autophagosomes (a double membrane organelle). This pathway has mainly been observed in the liver tissues of mammals and plant pollens [21,22]. To further inspect the potential lipophagy pathway occurring in castor bean germinating endosperm, we observed the morphological changes of LDs in endosperm throughout the seed germination under electron microscopy. As shown in Figure $6 \mathrm{~B}$, many LDs were directly bound with the vacuole surface and were eventually swallowed by vacuoles (Figure $6 \mathrm{Ba}-\mathrm{c}$ ) during seed germination. In particular, LDs that were swallowed by vacuoles were mainly observed after $4 \mathrm{DAG}$, which was associated with the expression of RcATGs in germinating endosperms. We did not, however, detect autophagosomes with a double-layer membrane structure. These observations indicated that the microlipophagy pathway mediated lipid degradation in germinating endosperm.

\section{Discussion}

There is increasing evidence that cell autophagy was extensively functionally involved in regulating plant development and responses to environmental stresses in a diverse set of plants [43,44]. As mentioned above, the castor bean has been considered a model material for studying seed biology in dicotyledonous plants [34-36]. Based on comprehensive analyses of identification and characterization of $A T G s$, we explored their putative functions in regulating seed development and germination in oilseed crop castor bean. To our knowledge, this is the first report on characterizing ATGs in the family Euphorbiaceae, which is an important group of plant resources. Here, we identified a total of 34 RcATGs that fall into four major cell autophagy processes, including the initial process-induced (ATG1/ATG13 kinase complex), membrane transport process (ATG9/2/18 complex), vesicle nucleation 
(PI3 kinase complex) and vesicle expansion. When inspecting the number of ATGs involved in the four conserved processes, we found 41 ATGs in maize [19], 32 in rice [17], 30 in tobacco [18], and only 12 in algae [41]. Variation in the number of ATGs might be related to the complexity and size of a plant's genome. These observations indicated that although the autophagy pathway is highly conserved, processes and biological functions (such as some specific physiological processes within different tissues) probably vary in different plants. Studies have found that ATG1s, ATG13s, ATG8s and ATG18s often have multiple copies in plants [45]. These multiple copies comprise their own subfamilies. Here, gene structure and phylogenetic analyses revealed that although there are unique and specific motifs, the gene copies were phylogenetically orthologous within each subfamily. Moreover, for ATG subfamilies (ATG1s, ATG13s, ATG8s and ATG18s), some unexpected associations were found among different plants in Figure 2, such as placing ZmATG18b and RcATG18b in Clade I while placing AtATG18b and OsATG18b in Clade II, and placing ZmATG13a and OsATG13a in Clade I while placing AtATG13a and RcATG18a in Clade II, which suggests the phylogeny of ATG subfamilies is complex.

Theoretically, the occurrence of the autophagy process requires the participation of multiple members within an activated autophagy network. A number of studies have shown that for a given member, its expressional profile varies among different tissues [19], implying that they might be involved in different biological processes. ATGs could be involved in regulating various processes of plant growth and development [43]. We identified differential expression of most ATGs in various tissues. We also noted that some ATGs were only expressed in specific castor bean tissues. The potential functions of these tissue-specific ATGs remain unknown in castor beans. In developing seeds, one of our main objectives was to explore the potential function of autophagy in regulating seed development. Our investigation found that many genes showed higher expression levels in the endosperm relative to the embryo. These ATGs that were highly expressed in the endosperm might be involved in regulating the metabolism and accumulation of storage materials [46]. Similarly, some atg mutants were previously found to cause a decrease in seed weight for Arabidopsis and maize [19,47]. These results mean that autophagy is involved in regulating storage material accumulation in seeds. In particular, our investigation found that most ATGs were highly expressed in the late stage of seed coat development, when the cell walls of seed coat cells experienced rapid lignification, resulting in hard and lignified seed coat tissues $[36,48]$. Previous studies had found that the programmed cell death caused by ricinosomes intensively occurred in the late stage of castor bean seed development (including the endosperm and seed coat tissues) [49,50]. Furthermore, the indicator gene CysEP for detecting the emergence of programmed cell death was highly up-regulated within the rapidly lignified castor bean seed coat tissues [50]. Thus, previous studies have confirmed that programmed cell death indeed occurs in seed coat tissues during the late stage of seed development in castor beans. However, the mechanism by which the process of programmed cell death was triggered remains unknown. Recent studies have revealed that autophagy is a critical factor in triggering programmed cell death [51,52]. Based on the intensive up-regulation of RcATGs in castor bean seed coat tissues, we infer that autophagy may be the trigger of programmed cell death during the late stage of castor bean seed coat development. It is further possible that autophagy is induced by programmed cell death for the clearance of terminally differentiated cells produced in the process of programmed cell death [53]. Many studies have found that $A T G$ s were up-regulated at the late stage of seed development in different plants, such as tobacco [18] and maize [19]. The role of autophagy in regulating seed maturation might be common, despite diversity in the types of plant seeds involved.

Another objective in our study was to explore the potential function of autophagy in regulating endosperm germination according to their expressional profiles in seed germination. The mature endosperm of castor bean seeds contains a large number of lipid droplets. These LDs are gradually decomposed during seed germination. For a long time, LDs were considered to be hydrolyzed in a lipolysis process by a series of lipases, such as ATGL (adipose triglyceride lipase), Tg13/Tg14 in yeast, HSL (hormone sensitive lipase) in mammals and GDSL lipases in plants [54,55]. After hydrolysis, the fatty acid product can provide energy to cells through $\beta$-oxidation in the mitochondria. Recent 
studies have shown that lipophagy is involved in the breakdown of LDs for the maintenance of lipid homeostasis [39]. One way in which lipophagy participates in degrading LDs is directly degrading them to fatty acids in the vacuoles, in lower organisms such as single cell algae (Auxenochlorella protothecoides) and yeast (S. cerevisiae), usually termed microlipophagy [41,42]. Another way in which lipophagy participates in degrading LDs is mediated by autophagosomes (a double membrane organelle). This is termed macrolipophagy, and has been observed by electron microscopy in plant pollen and in liver tissues of mammals [22,56]. Here, we observed degradation of LDs directly mediated by vacuoles and did not detect any double-membraned autophagosomes. The results of electron microscopy were similar to that of Auxenochlorella protothecoides and S. cerevisiae [41,42], suggesting that the role of autophagy in breaking down LDs might be largely mediated by the microlipophagy pathway in germinating castor bean endosperm. However, whether microlipophagy is a commonly used process in mediating the breakdown of LDs in other oilseeds remains unknown. Further research is required into microlipophagy in other oilseed species.

\section{Materials and Methods}

\subsection{Plant Materials}

The castor bean seeds var. ZB306 provided by Zibo Academy of Agricultural Science (Zibo, Shandong, China) were germinated and grown in the greenhouse under $13 \mathrm{~h} 28^{\circ} \mathrm{C}$ day and $11 \mathrm{~h} 22^{\circ} \mathrm{C}$ evening conditions. The root samples were collected at 14 DAG (days after germination) and the first internodes were collected as stems when grown to $5 \mathrm{~cm}$ in length. Leaves were collected two weeks after the blade appeared. Ovules were collected from the unfertilized fruits. Seed samples were collected from the greenhouse at different development stages from 10 to 50 days after pollination (DAP). Seed coats, endosperms and embryos were dissected after seeds were harvested, then immediately frozen in liquid nitrogen and stored at $-80^{\circ} \mathrm{C}$.

\subsection{Identification of ATGs in Castor Bean}

Based on the castor bean genome (http://castorbean.jcvi.org/index.php), an extensive search was performed to identify all ATG genes in castor beans. The amino acid sequences of ATG genes generically are rather conserved with specific domains; therefore, the known ATG genes of Arabidopsis [23-26] could be used as queries for globally searching ATG genes in a given plant species. First, to identify the putative ATGs in castor beans, the BLASTP search was performed using the known Arabidopsis ATG protein sequences downloaded from the TAIR (https://www.arabidopsis.org/), as queries against the castor bean genome database. Genes with a significant E-value $\left(<10^{-5}\right)$ were collected while the redundant genes were discarded from our data set. After that, the online programs SMART (http://smart.embl-heidelberg.de/) and pfam (http://pfam.sanger.ac.uk/) were used to check the predicted ATG domains in these collected proteins. The genes which showed the most significant E-value with ATG domains were considered as putative ATGs of castor beans (RcATGs).

\subsection{Bioinformatic Analysis and Phylogenetic Construction}

The molecular weight (MW) and theoretical isoelectric point (PI) of different proteins were predicted using the Compute pI/Mw tool-ExPASy (http://web.expasy.org/compute_pi/). Intron/extron structure were analyzed using the online tool-GSDS 2.0 (http://gsds.cbi.pku.edu.cn/) based on genomic DNA sequences and the CDS sequences. Conserved motifs in RcATGs were identified by MEME online software (http://meme-suite.org/tools/meme) with the following parameters: normal motif discovery, optimum motif width (10-200 amino acids), 20 for maximum number of motifs and zero or one occurrence per sequence. For phylogenetic tree construction, amino acid sequences of ATGs from different species including Arabidopsis, rice, tobacco, corn, yeast were aligned using Clustal $\mathrm{X}$ version 2.1 first and then phylogenetic trees were constructed using MEGA X [57] by the neighbor-joining method with 1000 bootstrap replicates. 


\subsection{Analysis of RNA-seq Data}

To investigate the expression profile of RcATGs in different tissues, the FPKM values of RcATGs in different tissues including root, stem, leaf, seedling, ovule, capsule, inflorescence and seed of different development stages (10 DAP for seed1; 20 DAP for seed2; 30 DAP for seed3; 40 DAP for seed4; 50 DAP for seed5) were acquired from the castor bean genome database (https://woodyoilplants.iflora.cn/). The pheatmap R package was used to map the heatmap and each RcATG was normalized to a Z-score based on FPKM values. Based on the 'complete' clustering method, hierarchical cluster analysis was performed.

\subsection{RNA Extraction and $q P C R$}

Total RNA was isolated from different tissues using RNAiso (TaKaRa). High quality RNA (three bands on agarose gel, absorbance ratio 260/230 1.8-2.0) were used to synthesize the cDNA with TransScript All-in-One First-Strand cDNA Synthesis SuperMix for qPCR (Trangene) following the procedure recommended by the manufacturer. The qPCR was carried out in a $20 \mu \mathrm{L}$ PCR mixture containing $10 \mu \mathrm{L} 2 \mathrm{X}$ TransStart Tip Green qPCR SuperMix (Transgene), $0.4 \mu \mathrm{L}$ forward primer $(10 \mu \mathrm{M})$, $0.4 \mu \mathrm{L}$ reverse primer $(10 \mu \mathrm{M}), 1 \mathrm{ng}$ cDNA prepared from different materials and variable $\mathrm{ddH}_{2} \mathrm{O}$. The RcACTIN2 was used as a control to normalize different samples. The specific PCR procedures are described as follows: denaturation at $94{ }^{\circ} \mathrm{C}$ for $30 \mathrm{~s}$; then 45 cycles of $5 \mathrm{~s}$ denaturation at $94{ }^{\circ} \mathrm{C}$ and $30 \mathrm{~s}$ of annealing and synthesis at $60^{\circ} \mathrm{C}$. The primers for qPCR were designed using software primer premier 5 to ensure the specificity of amplified product, and are shown in Table S1. All assays were performed at least three times from three biological replicates. The pheatmap $\mathrm{R}$ package was used to map the heatmap and each RcATG was normalized to a Z-score depending on the fold change.

\subsection{Determination of Lipid Content}

The method used for total lipid extraction was previously described by Xu et al. [58]. After grinding with liquid nitrogen for a few minutes, a fixed weight of dried sample (W) was added to $4 \mathrm{~mL}$ extracting solution (hexane/isopropanol $=3: 2)(v / v)$. The mixture was fully shaken and then the supernatant was collected after centrifugation at $5000 \times g$ for $5 \mathrm{~min}$. The precipitation was mixed with $4 \mathrm{~mL}$ extracting solution, and then the supernatant was collected again after centrifugation at $5000 \times g$ for $5 \mathrm{~min}$. The combined supernatant was vacuum dried at a pressure of $-60 \mathrm{kPa}$ at $50{ }^{\circ} \mathrm{C}$ to a constant weight $(\mathrm{w})$. The lipid content was equal to $\mathrm{w} / \mathrm{W} \times 100 \%$. All assays were performed on at least three biological replicates.

\subsection{Transmission Electron Microscopy}

The castor bean seeds were cut into small pieces and fixed in a $0.05 \mathrm{M}$ cacodylate buffer (pH 7.4) containing $2 \%$ glutaraldehyde and $4 \%$ paraformaldehyde for $12 \mathrm{~h}$ in an ice bath. The electron microscopy sections were prepared as described by Jiang et al. [59]. Cell images were taken using a transmission electron microscope (JEM-1200EX2, JEOL).

Supplementary Materials: The following are available online at http://www.mdpi.com/1422-0067/21/2/562/s1.

Author Contributions: Conceived and designed the experiments: B.H. and A.L. Analyzed the data: B.H. and W.X. Collection of tissues and electron microscopic observation: B.H. and Y.F. RT-qPCR: H.X. Wrote the paper: B.H., Q.C. and A.L. All authors have read and agreed to the published version of the manuscript.

Funding: This work was partially supported by National Natural Science Foundation of China (31661143002, 81760507, 31571709, 31771839, 31701123 and 31501034), Yunnan Applied Basic Research Projects (2016FA011, 2016FB060 and 2016FB040), the National R\&D Infrastructure and Facility development Program of China "Fundamental Science Data Sharing Platform (DKA2017-12-02-16) and the $13^{\text {th }}$ Five-year informatization Plan of Chinese Academy of Sciences (No. XXH13506).

Acknowledgments: We thank the Service Center for Experimental Biotechnology in the Key Laboratory of Economic Plants and Biotechnology, Kunming Institute of Botany, Chinese Academy of Sciences, for assistance with the qPCR analysis. 
Conflicts of Interest: The authors declare no conflict of interest.

\section{References}

1. Reggiori, F.; Klionsky, D.J. Autophagy in the eukaryotic cell. Eukaryot. Cell 2002, 1, 11-21. [CrossRef]

2. Yang, Z.; Klionsky, D.J. An overview of the molecular mechanism of autophagy. Curr. Top. Microbiol. Immunol. 2009, 335, 1-32.

3. Thompson, A.R.; Vierstra, R.D. Autophagic recycling: Lessons from yeast help define the process in plants. Curr. Opin. Plant Biol. 2005, 8, 165-173. [CrossRef]

4. Klionsky, D.J.; Meijer, A.J.; Codogno, P. Autophagy and p70S6 kinase. Autophagy 2005, 1, 59-60, discussion 60-1. [CrossRef]

5. Wen, X.; Klionsky, D.J. An overview of macroautophagy in yeast. J. Mol. Biol. 2016, 428, 1681-1699. [CrossRef]

6. Li, W.W.; Li, J.; Bao, J.K. Microautophagy: Lesser-known self-eating. Cell. Mol. Life Sci. CMLS 2012, 69, 1125-1136. [CrossRef]

7. Wang, P.; Mugume, Y.; Bassham, D.C. New advances in autophagy in plants: Regulation, selectivity and function. Semin. Cell Dev. Biol. 2018, 80, 113-122. [CrossRef] [PubMed]

8. Slavikova, S.; Shy, G.; Yao, Y.; Glozman, R.; Levanony, H.; Pietrokovski, S.; Elazar, Z.; Galili, G. The autophagy-associated Atg8 gene family operates both under favourable growth conditions and under starvation stresses in Arabidopsis plants. J. Exp. Bot. 2005, 56, 2839-2849. [CrossRef]

9. Xiong, Y.; Contento, A.L.; Bassham, D.C. AtATG18a is required for the formation of autophagosomes during nutrient stress and senescence in Arabidopsis thaliana. Plant J. 2005, 42, 535-546. [CrossRef]

10. Sheng, X.Y.; Wei, Q.; Jiang, L.P.; Li, X.; Gao, Y.; Wang, L. Different Degree in Proteasome Malfunction Has Various Effects on Root Growth Possibly through Preventing Cell Division and Promoting Autophagic Vacuolization. PLoS ONE 2012, 7, e45673. [CrossRef]

11. Sankaranarayanan, S.; Samuel, M.A. A proposed role for selective autophagy in regulating auxin-dependent lateral root development under phosphate starvation in Arabidopsis. Plant Signal. Behav. 2015, 10, e989749. [CrossRef]

12. Ohsumi, Y. Historical landmarks of autophagy research. Cell Res. 2014, 24, 9-23. [CrossRef]

13. Qin, G.; Ma, Z.; Zhang, L.; Xing, S.; Hou, X.; Deng, J.; Liu, J.; Chen, Z.; Qu, L.J.; Gu, H. Arabidopsis AtBECLIN 1/AtAtg6/AtVps30 is essential for pollen germination and plant development. Cell Res. 2007, 17, $249-263$. [CrossRef]

14. Xu, N.; Gao, X.Q.; Zhao, X.Y.; Zhu, D.Z.; Zhou, L.Z.; Zhang, X.S. Arabidopsis AtVPS15 is essential for pollen development and germination through modulating phosphatidylinositol 3-phosphate formation. Plant Mol. Biol. 2011, 77, 251-260. [CrossRef]

15. Rose, T.L.; Bonneau, L.; Der, C.; Marty-Mazars, D.; Marty, F. Starvation-induced expression of autophagy-related genes in Arabidopsis. Biol. Cell 2006, 98, 53-67. [CrossRef]

16. Guiboileau, A.; Yoshimoto, K.; Soulay, F.; Bataille, M.P.; Avice, J.C.; Masclaux-Daubresse, C. Autophagy machinery controls nitrogen remobilization at the whole-plant level under both limiting and ample nitrate conditions in Arabidopsis. New Phytol. 2012, 194, 732-740. [CrossRef]

17. Wada, S.; Hayashida, Y.; Izumi, M.; Kurusu, T.; Hanamata, S.; Kanno, K.; Kojima, S.; Yamaya, T.; Kuchitsu, K.; Makino, A.; et al. Autophagy Supports Biomass Production and Nitrogen Use Efficiency at the Vegetative Stage in Rice. Plant Physiol. 2015, 168, 60-73. [CrossRef]

18. Zhou, X.M.; Zhao, P.; Wang, W.; Zou, J.; Cheng, T.H.; Peng, X.B.; Sun, M.X. A comprehensive, genome-wide analysis of autophagy-related genes identified in tobacco suggests a central role of autophagy in plant response to various environmental cues. DNA Res. Int. J. Rapid Publ. Rep. Genes Genomes 2015, 22, 245-257. [CrossRef]

19. Li, F.; Chung, T.; Pennington, J.G.; Federico, M.L.; Kaeppler, H.F.; Kaeppler, S.M.; Otegui, M.S.; Vierstra, R.D. Autophagic recycling plays a central role in maize nitrogen remobilization. Plant Cell 2015, 27, 1389-1408. [CrossRef] [PubMed]

20. Lapierre, L.R.; Silvestrini, M.J.; Nunez, L.; Ames, K.; Wong, S.; Le, T.T.; Hansen, M.; Melendez, A. Autophagy genes are required for normal lipid levels in C. elegans. Autophagy 2013, 9, 278-286. [CrossRef]

21. Martinez-Lopez, N.; Singh, R. Autophagy and Lipid Droplets in the Liver. Annu. Rev. Nutr. 2015, 35, $215-237$. [CrossRef] 
22. Kurusu, T.; Koyano, T.; Hanamata, S.; Kubo, T.; Noguchi, Y.; Yagi, C.; Nagata, N.; Yamamoto, T.; Ohnishi, T.; Okazaki, Y.; et al. OsATG7 is required for autophagy-dependent lipid metabolism in rice postmeiotic anther development. Autophagy 2014, 10, 878-888. [CrossRef]

23. Thompson, A.R.; Doelling, J.H.; Suttangkakul, A.; Vierstra, R.D. Autophagic nutrient recycling in Arabidopsis directed by the ATG8 and ATG12 conjugation pathways. Plant Physiol. 2005, 138, 2097-2110. [CrossRef]

24. Bassham, D.C.; Laporte, M.; Marty, F.; Moriyasu, Y.; Ohsumi, Y.; Olsen, L.J.; Yoshimoto, K. Autophagy in development and stress responses of plants. Autophagy 2006, 2, 2-11. [CrossRef]

25. Seay, M.D.; Patel, S.; Dinesh-Kumar, S.P. Autophagy and plant innate immunity. Cell. Microbiol. 2006, 8, 899-906. [CrossRef] [PubMed]

26. Bassham, D.C. Plant autophagy-more than a starvation response. Curr. Opin. Plant Biol. 2007, 10, 587-593. [CrossRef]

27. Xia, K.; Liu, T.; Ouyang, J.; Wang, R.; Fan, T.; Zhang, M. Genome-wide identification, classification, and expression analysis of autophagy-associated gene homologues in rice (Oryza sativa L.). DNA Res. Int. J. Rapid Publ. Rep. Genes Genomes 2011, 18, 363-377. [CrossRef]

28. Zhai, Y.F.; Guo, M.; Wang, H.; Lu, J.P.; Liu, J.H.; Zhang, C.; Gong, Z.H.; Lu, M.H. Autophagy, a Conserved Mechanism for Protein Degradation, Responds to Heat, and Other Abiotic Stresses in Capsicum annuum L. Front. Plant Sci. 2016, 7, 131. [CrossRef]

29. Shangguan, L.; Fang, X.; Chen, L.; Cui, L.; Fang, J. Genome-wide analysis of autophagy-related genes (ARGs) in grapevine and plant tolerance to copper stress. Planta 2018, 247, 1449-1463. [CrossRef]

30. De Souza Costa, E.T.; Guilherme, L.R.; De Melo, E.E.; Ribeiro, B.T.; Dos Santos, B.I.E.; Da Costa Severiano, E.; Faquin, V.; Hale, B.A. Assessing the tolerance of castor bean to $\mathrm{Cd}$ and $\mathrm{Pb}$ for phytoremediation purposes. Biol. Trace Elem. Res. 2012, 145, 93-100. [CrossRef]

31. Patel, V.R.; Dumancas, G.G.; Kasi Viswanath, L.C.; Maples, R.; Subong, B.J. Castor Oil: Properties, Uses, and Optimization of Processing Parameters in Commercial Production. Lipid Insights 2016, 9, 1-12. [CrossRef]

32. Ogunniyi, D.S. Castor oil: A vital industrial raw material. Bioresour. Technol. 2006, 97, 1086-1091. [CrossRef]

33. Greenwood, J.S.; Bewley, J.D. Seed Development In Ricinus-Communis Cv Hale (Castor Bean) .3. Pattern Of Storage Protein And Phytin Accumulation In the Endosperm. Can. J. Bot. 1985, 63, 2121-2128. [CrossRef]

34. Houston, N.L.; Hajduch, M.; Thelen, J.J. Quantitative Proteomics of Seed Filling in Castor: Comparison with Soybean and Rapeseed Reveals Differences between Photosynthetic and Nonphotosynthetic Seed Metabolism. Plant Physiol. 2009, 151, 857-868. [CrossRef]

35. Nogueira, F.C.S.; Palmisano, G.; Schwammle, V.; Campos, F.A.P.; Larsen, M.R.; Domont, G.B.; Roepstorff, P. Performance of Isobaric and Isotopic Labeling in Quantitative Plant Proteomics. J. Proteome Res. 2012, 11, 3046-3052. [CrossRef]

36. Xu, W.; Dai, M.Y.; Li, F.; Liu, A.Z. Genomic imprinting, methylation and parent-of-origin effects in reciprocal hybrid endosperm of castor bean. Nucleic Acids Res. 2014, 42, 6987-6998. [CrossRef]

37. Chan, A.P.; Crabtree, J.; Zhao, Q.; Lorenzi, H.; Orvis, J.; Puiu, D.; Melake-Berhan, A.; Jones, K.M.; Redman, J.; Chen, G.; et al. Draft genome sequence of the oilseed species Ricinus communis. Nat. Biotechnol. 2010, 28, 951-956. [CrossRef]

38. Wan, L.L.; Xia, Q.; Qiu, X.; Selvaraj, G. Early stages of seed development in Brassica napus: A seed coat-specific cysteine proteinase associated with programmed cell death of the inner integument. Plant J. 2002, 30, 1-10. [CrossRef]

39. Liu, K.; Czaja, M.J. Regulation of lipid stores and metabolism by lipophagy. Cell Death Differ. 2013, $20,3-11$. [CrossRef]

40. Elander, P.H.; Minina, E.A.; Bozhkov, P.V. Autophagy in turnover of lipid stores: Trans-kingdom comparison. J. Exp. Bot. 2018, 69, 1301-1311. [CrossRef]

41. Zhao, L.; Dai, J.; Wu, Q. Autophagy-like processes are involved in lipid droplet degradation in Auxenochlorella protothecoides during the heterotrophy-autotrophy transition. Front. Plant Sci. 2014, 5, 400. [CrossRef]

42. Van Zutphen, T.; Todde, V.; De Boer, R.; Kreim, M.; Hofbauer, H.F.; Wolinski, H.; Veenhuis, M.; Van der Klei, I.J.; Kohlwein, S.D. Lipid droplet autophagy in the yeast Saccharomyces cerevisiae. Mol. Biol. Cell 2014, 25, 290-301. [CrossRef]

43. Batoko, H.; Dagdas, Y.; Baluska, F.; Sirko, A. Understanding and exploiting autophagy signaling in plants. Essays Biochem. 2017, 61, 675-685. 
44. Ustun, S.; Hafren, A.; Hofius, D. Autophagy as a mediator of life and death in plants. Curr. Opin. Plant Biol. 2017, 40, 122-130. [CrossRef]

45. Il Kwon, S.; Park, O.K. Autophagy in Plants. J. Plant Biol. 2008, 51, 313-320. [CrossRef]

46. Pottier, M.; Masclaux-Daubresse, C.; Yoshimoto, K.; Thomine, S. Autophagy as a possible mechanism for micronutrient remobilization from leaves to seeds. Front. Plant Sci. 2014, 5, 11. [CrossRef] [PubMed]

47. Di Berardino, J.; Marmagne, A.; Berger, A.; Yoshimoto, K.; Cueff, G.; Chardon, F.; Masclaux-Daubresse, C.; Reisdorf-Cren, M. Autophagy controls resource allocation and protein storage accumulation in Arabidopsis seeds. J. Exp. Bot. 2018, 69, 1403-1414. [CrossRef] [PubMed]

48. Raviv, B.; Aghajanyan, L.; Granot, G.; Makover, V.; Frenkel, O.; Gutterman, Y.; Grafi, G. The dead seed coat functions as a long-term storage for active hydrolytic enzymes. PLoS ONE 2017, 12, e0181102. [CrossRef]

49. Schmid, M.; Simpson, D.; Kalousek, F.; Gietl, C. A cysteine endopeptidase with a C-terminal KDEL motif isolated from castor bean endosperm is a marker enzyme for the ricinosome, a putative lytic compartment. Planta 1998, 206, 466-475. [CrossRef]

50. Schmid, M.; Simpson, D.J.; Sarioglu, H.; Lottspeich, F.; Gietl, C. The ricinosomes of senescing plant tissue bud from the endoplasmic reticulum. Proc. Natl. Acad. Sci. USA 2001, 98, 5353-5358. [CrossRef] [PubMed]

51. Kurusu, T.; Kuchitsu, K. Autophagy, programmed cell death and reactive oxygen species in sexual reproduction in plants. J. Plant Res. 2017, 130, 491-499. [CrossRef] [PubMed]

52. Wang, H.B.; Lu, Q.; Cheng, S.Y.; Wang, X.C.; Zhang, H. Autophagy activity contributes to programmed cell death in Caenorhabditis elegans. Autophagy 2013, 9, 1975-1982. [CrossRef] [PubMed]

53. Minina, E.A.; Bozhkov, P.V.; Hofius, D. Autophagy as initiator or executioner of cell death. Trends Plant Sci. 2014, 19, 692-697. [CrossRef]

54. Toyoshi, F.; Parton, R.G. Not just fat: The structure and function of the lipid droplet. Cold Spring Harb. Perspect. Biol. 2011, 3, a004838.

55. Gao, M.J.; Yi, X.; Yang, W.B.; Lam, S.M.; Tong, X.H.; Liu, J.Y.; Wang, X.; Li, Q.; Shui, G.H.; He, Z.H. GDSL lipases modulate immunity through lipid homeostasis in rice. PLoS Pathog. 2017, 13, e1006724. [CrossRef]

56. Singh, R.; Kaushik, S.; Wang, Y.J.; Xiang, Y.Q.; Novak, I.; Komatsu, M.; Tanaka, K.; Cuervo, A.M.; Czaja, M.J. Autophagy regulates lipid metabolism. Nature 2009, 458, 1131-1135. [CrossRef]

57. Kumar, S.; Stecher, G.; Li, M.; Knyaz, C.; Tamura, K. MEGA X: Molecular Evolutionary Genetics Analysis across Computing Platforms. Mol. Biol. Evol. 2018, 35, 1547-1549. [CrossRef]

58. Xu, R.H.; Wang, R.L.; Liu, A.Z. Expression profiles of genes involved in fatty acid and triacylglycerol synthesis in developing seeds of Jatropha (Jatropha curcas L.). Biomass Bioenerg. 2011, 35, 1683-1692. [CrossRef]

59. Jiang, Q.; Zhao, L.; Dai, J.B.; Wu, Q.Y. Analysis of Autophagy Genes in Microalgae: Chlorella as a Potential Model to Study Mechanism of Autophagy. PLoS ONE 2012, 7, e41826. [CrossRef] [PubMed]

(C) 2020 by the authors. Licensee MDPI, Basel, Switzerland. This article is an open access article distributed under the terms and conditions of the Creative Commons Attribution (CC BY) license (http://creativecommons.org/licenses/by/4.0/). 